\title{
FEASIBILITY AND SAFETY OF FLUOROLESS URETERORENOSCOPY- OUR INSTITUTIONAL EXPERIENCE
}

\author{
Balaji Appiya Ramamoorthy1, Sangameswaran Palanisamy²
}

1 Professor and HOD, Department of Urology, Government Stanley Medical College affiliated to Tamilnadu Dr. MGR Medical University. ${ }^{2}$ Postgraduate Student, Department of Urology, Government Stanley Medical College affiliated to Tamilnadu Dr. MGR Medical University.

\section{ABSTRACT}

\section{BACKGROUND}

Patients with urolithiasis are exposed to significant amounts of radiation during preoperative workup by means of X-ray, conventional contrast imaging and CECT during surgery and followup. The purpose of this study was to determine the feasibility of performing ureterorenoscopy without fluoroscopic aid. In addition, we compared this technique with conventional fluoroscopic ureterorenoscopy.

\section{MATERIALS AND METHODS}

This is a retrospective analysis of hospital records based study in which 50 patients of mid and lower ureteric calculus undergoing fluoroless ureterorenoscope in our institution from August 2015 to December 2016 were reviewed retrospectively. These procedures were performed safely by inserting guide wires and instruments using tactile feedback, direct visualisation, and external visual cues to substitute for fluoroscopy. Single shot X-ray was done at the end to confirm the position of stent. In addition, this cohort was compared with 50 conventional, fluoroscopy-guided ureterorenoscopies performed in the same time period.

\section{RESULTS}

Fifty ureterorenoscopies were performed without c-arm guidance. In these group of patients, the mean operative time was 57.30 minutes, the mean stone burden was $61.1 \mathrm{~mm}^{2}$, complication rate was $6 \%$, and repeat procedure rate was $7 \%$. Compared with conventional ureterorenoscopy, the fluoroless ureterorenoscopy patients had a similar age, gender, body-mass index, operative time but lesser mean stone burden (61.16 vs. $66.58 \mathrm{~mm}^{2}$ ) and lesser repeat procedure rate.

\section{CONCLUSION}

This study demonstrates the feasibility, safety and efficacy of the completely fluoroless ureterorenoscopic treatment of mid and lower ureteric calculi while completely avoiding radiation exposure to the patients and staff. Apart from its usefulness in high risk patients like pregnant women, children and recurrent calculus patients, this fluoroless technique offers an alternative for reduction of radiation in all patients.

\section{KEYWORDS}

Fluoroless, Ureterorenoscopy, Radiation Exposure.

HOW TO CITE THIS ARTICLE: Ramamoorthy BA, Palanisamy S. Feasibility and safety of fluoroless ureterorenoscopy- Our institutional experience. J. Evolution Med. Dent. Sci. 2017;6(53):4007-4011, DOI: 10.14260/Jemds/2017/866

\section{BACKGROUND}

Radiological diagnostic imaging has been an integral part of day-to-day urology practice. Due to advances in imaging techniques, there has also been new insight into the deleterious effects of radiation exposure. ${ }^{1}$ There has been a heightened awareness of the negative effects of medical imaging that has prompted medical professionals to reevaluate their current practice regarding radiation to their patients and themselves.

Financial or Other, Competing Interest: None.

Submission 06-04-2017, Peer Review 21-06-2017,

Acceptance 27-06-2017, Published 03-07-2017.

Corresponding Author:

Dr. Balaji Appiya Ramamoorthy,

Professor and HOD,

Department of Urology,

NSB 6th Floor,

Government Stanley Medical College,

Old Jail Road,

Royapuram, Mint.

Chennai 600001

E-mail:drcps03@gmail.com

DOI: $10.14260 /$ jemds $/ 2017 / 866$
Fluoroscopic imaging routinely plays an integral role during ureterorenoscopic lithotripsy. It is commonly used to identify stones, assist in renal mapping, and verify placement of guide wires, stents, and ureteral sheaths. A limitation of fluoroscopy is the potentially significant radiation exposure to the patient, physician, and operating room staff. There is no lower limit of radiation exposure below which potentially harmful biologic effects do not occur; therefore, any exposure has the possibility to induce malignancy.

All ionising radiation carries a risk to cause cancer. ${ }^{2}$ In order to reduce the risk, we follow the procedure of doing ureterorenoscopy without fluoroscopy. The purpose of this study was to determine the safety and feasibility of ureterorenoscopy without image guidance and to compare outcomes of fluoroless ureterorenoscopy with a cohort of conventional ureterorenoscopy patients performed using fluoroscopy.

\section{MATERIALS AND METHODS}

The study is a retrospective analysis of hospital records based study comparing two groups of patients. Between August 2015 and December 2016, 50 cases of simple ureterorenoscopic lithotripsy of ureteral stones performed at our institution, 
without the use of fluoroscopy or ultrasound, were retrospectively evaluated and compared with routine fluoroscopy guided ureteroscopy procedure. Cases of malignancy, duplicated collecting systems, previously documented strictures, upper ureteric and renal calculus and treatment of non-urolithiasis-related conditions were excluded from the study. Variables compared are age at time of surgery, gender, laterality, body-mass index (BMI), presence of preoperative stent, stone location, and mean operative time. Stone size was calculated by multiplying the two largest cross-sectional dimensions to obtain a stone area in $\mathrm{mm}^{2}$. Outcomes analysed included complication rates, stone-free rates (defined as no residual stone $>4 \mathrm{~mm}$ ), and repeat procedure rates. We then compared these patients with a similar cohort of 50 patients who underwent ureterorenoscopy for urolithiasis with conventional fluoroscopic guidance in our institution in the same time period. The same demographic, perioperative information, and outcome measures were collected. Groups were compared with a Student's t-test for continuous variables and Fisher's exact test for categorical variables. Significance was set at $\mathrm{p}<0.05$.

\section{Technique for Fluoroless Ureterorenoscopy}

The procedure begins with a thorough assessment of preoperative imaging. Under spinal anaesthesia, the patient was placed in the lithotomy position. A semirigid wolf ureterorenoscopy of $6 \mathrm{Fr}$ and/or $8 \mathrm{Fr}$ are used for lithotripsy. After locating the desired ureteral orifice, under glide wire guidance, ureteric orifice entered. If a previous stent was in place, this wire was placed beside the existing stent before removal. Using tactile feedback, the glide wire was gently manipulated proximally until the resistance of the stone could be felt. With gentle back and forth movements, the wire was advanced beyond the stone and up until resistance from contact with the kidney was encountered. Using tactile manipulation, the semirigid ureterorenoscope was advanced under direct vision until the stone was encountered. During ureterorenoscope insertion, the two main locations where resistance that provides tactile feedback occurs are when the ureterorenoscope passes the prostate/bladder neck and the ureteral orifice. The ureterorenoscope was advanced beyond the ureteral orifice while the assistant held the glide wire taut. Once the stone is visualised, stone is broken with glide wire in situ or removed depending on ureterorenoscope size (8-Fr/6Fr).

Following completion of stone treatment, a 5-Fr/4-Fr 26 $\mathrm{cm}$ stent was placed in all patients using a PTFE guide wire. We then pulled the ureterorenoscope back to the ureterovesical junction (UVJ) while inspecting the ureter to evaluate mucosal integrity and assess for residual stones. If the ureterorenoscope would not pass up to the UPJ, the ureteral length could be estimated using the preoperative CT. A 6-F multi-length stent was then advanced proximally over the safety wire into the ureter under direct visualisation. After advancing the stent proximally to the appropriate ureteral distance, the guide wire was pulled back 10 to $15 \mathrm{~cm}$ and the stent was then advanced under direct vision within the bladder until the distal end was at the mid bladder neck. The wire was removed and a proper bladder coil was endoscopically confirmed. At any point during the procedure, if excessive resistance was encountered during guide wire or ureter or endoscope insertion, if wire lengths were not appropriate following placement, or if a tightly impacted stone was not amenable to passing the guide wire, then an ultra-lowdose fluoroscopy-guided technique was used.

\section{RESULTS}

Between 2015 and 2016, 50 ureterorenoscopies to treat urolithiasis were performed without intraoperative image guidance and the results are compared here.

Totally 100 cases were analysed. Out of which $50(n=50)$ were conventional fluoroscopic URSL (group 1) and $50(n=50)$ were fluoroless (group 2) procedures. The mean age of group 1 was 40.64 and group 2 was 40.52 . The mean stone area was 66.58 and $61.16 \mathrm{~mm}^{2}$ in group $1 \& 2$ respectively. The mean operative time was $59.52 \mathrm{~min}$. in group 1 and $57.30 \mathrm{~min}$. in group 2. Mean fluoro time in group 1 was $35.64 \mathrm{sec}$. (Table 1). Age, stone area and mean operative time are independent variables. Except for fluoro time, there was no difference in both groups. In both groups, stone clearance rate was $92 \%$ (Table 2). The postoperative complication (Steinstrasse, stent symptoms) rate was $6 \%$ in both groups (Table 3 ). $8 \%$ of group 1 patients and $6 \%$ of group 2 have undergone repeat procedure (Table 4). When compared with conventional ureterorenoscopy with image guidance, there was no statistical difference in age, BMI, stone clearance rate, complication rate, operative time, re-procedure rate. Logistic regression multivariate analysis was performed with all study parameters in relation to the repeat procedure rate. It was found that operative time was significantly predictive of repeat procedures in both groups probably because of increased time requirement for clearance of impacted stone. There was no association between the fluoroless technique and gender, laterality or BMI. It is clear from our study that the main advantage of fluoroless technique is that there is no radiation exposure for operating surgeons and assisting staff.

\begin{tabular}{|c|c|c|c|c|c|}
\hline & Procedure & N & Mean & Std. Deviation & Std. Error Mean \\
\hline \multirow{2}{*}{ Age } & Group 1 & 50 & 40.64 & 14.327 & 2.026 \\
\cline { 2 - 6 } & Group 2 & 50 & 40.52 & 14.194 & 2.007 \\
\hline \multirow{2}{*}{ Stone area } & Group 1 & 50 & 66.5886 & 56.53281 & 7.99495 \\
\cline { 2 - 6 } & Group 2 & 50 & 61.1688 & 37.22498 & 5.26441 \\
\hline \multirow{2}{*}{ Fluoro time (Sec.) } & Group 1 & 50 & 35.64 & 9.499 & 1.343 \\
\cline { 2 - 6 } Mean operative time (min.) & Group 2 & $0^{\mathrm{a}}$ &. &. & 2.454 \\
\cline { 2 - 6 } & Group 1 & 50 & 59.52 & 17.354 & 2.426 \\
\hline
\end{tabular}




\begin{tabular}{|c|c|c|c|c|c|}
\hline & & & \multicolumn{2}{|c|}{$\begin{array}{l}\text { Stone Free Rate } \\
\end{array}$} & \multirow{2}{*}{ Total } \\
\hline & & & Complete & Incomplete & \\
\hline \multirow{6}{*}{ Procedure } & \multirow{3}{*}{ Group 1} & Count & 46 & 4 & 50 \\
\hline & & Expected Count & 46.0 & 4.0 & 50.0 \\
\hline & & $\%$ within Procedure & $92.0 \%$ & $8.0 \%$ & $100.0 \%$ \\
\hline & \multirow{3}{*}{ Group 2} & Count & 46 & 4 & 50 \\
\hline & & Expected Count & 46.0 & 4.0 & 50.0 \\
\hline & & $\%$ within Procedure & $92.0 \%$ & $8.0 \%$ & $100.0 \%$ \\
\hline \multirow{3}{*}{\multicolumn{2}{|c|}{ Total }} & Count & 92 & 8 & 100 \\
\hline & & Expected Count & 92.0 & 8.0 & 100.0 \\
\hline & & $\%$ within Procedure & $92.0 \%$ & $8.0 \%$ & $100.0 \%$ \\
\hline
\end{tabular}

\begin{tabular}{|c|c|c|c|c|c|}
\hline & & & \multicolumn{2}{|c|}{ Post-op Complications } & \multirow{2}{*}{ Total } \\
\hline & & & No & Yes & \\
\hline \multirow{6}{*}{ Procedure } & \multirow{3}{*}{ Group 1} & Count & 47 & 3 & 50 \\
\hline & & Expected Count & 47.0 & 3.0 & 50.0 \\
\hline & & \% within Procedure & $94.0 \%$ & $6.0 \%$ & $100.0 \%$ \\
\hline & \multirow{3}{*}{ Group 2} & Count & 47 & 3 & 50 \\
\hline & & Expected Count & 47.0 & 3.0 & 50.0 \\
\hline & & \% within Procedure & $94.0 \%$ & $6.0 \%$ & $100.0 \%$ \\
\hline \multirow{3}{*}{\multicolumn{2}{|c|}{ Total }} & Count & 94 & 6 & 100 \\
\hline & & Expected Count & 94.0 & 6.0 & 100.0 \\
\hline & & \% within Procedure & $94.0 \%$ & $6.0 \%$ & $100.0 \%$ \\
\hline \multicolumn{6}{|c|}{ Table 3. Procedure * Post-op Complications } \\
\hline
\end{tabular}

\begin{tabular}{|c|c|c|c|c|c|}
\hline & & & \multicolumn{2}{|c|}{ Repeat Procedures } & \multirow{2}{*}{ Total } \\
\hline & & & No & Yes & \\
\hline \multirow{6}{*}{ Procedure } & \multirow{3}{*}{ Group 1} & Count & 46 & 4 & 50 \\
\hline & & Expected Count & 46.5 & 3.5 & 50.0 \\
\hline & & $\%$ within Procedure & $92.0 \%$ & $8.0 \%$ & $100.0 \%$ \\
\hline & \multirow{3}{*}{ Group 2} & Count & 47 & 3 & 50 \\
\hline & & Expected Count & 46.5 & 3.5 & 50.0 \\
\hline & & $\%$ within Procedure & $94.0 \%$ & $6.0 \%$ & $100.0 \%$ \\
\hline \multirow{3}{*}{\multicolumn{2}{|c|}{ Total }} & Count & 93 & 7 & 100 \\
\hline & & Expected Count & 93.0 & 7.0 & 100.0 \\
\hline & & \% within Procedure & $93.0 \%$ & $7.0 \%$ & $100.0 \%$ \\
\hline \multicolumn{6}{|c|}{ Table 4. Procedure ${ }^{*}$ Repeat Procedures } \\
\hline
\end{tabular}

\section{DISCUSSION}

The prevalence of urinary calculi is $2 \%$ to $3 \%$ in the general population, and over a lifetime, urinary calculi are expected to afflict greater than $12 \%$ of men and $6 \%$ of women with a recurrence rate of $30 \%$ to $40 \%$ within 5 years. The prevalence of urinary calculi appears to be increasing. Radiographic imaging is necessary to confirm or exclude urinary calculi, and those patients with recurrent abdominal or flank pain are at risk for multiple doses of diagnostic radiation. Furthermore, this dose effect is increased when intervention is warranted. Although the doses are not routinely high enough to cause the deterministic effects of radiation such as skin burns, the stochastic effects of radiation may result in the creation of malignancy. Certainly, the reduction of the radiation exposure will reduce the risk of radiation-associated morbidity. Fluoroscopy is routinely employed to guide the surgeon during upper tract stone treatment. Performing the procedure with reduced amounts of fluoroscopy represents a significant departure from the conventional endoscopic technique. In the field of Interventional Cardiology, Georges et $\mathrm{al}^{3}$ reported a
$50 \%$ reduction in radiation exposure with a 15-hour educational course and standardised radiation reduction technical recommendations. This fluoroless ureterorenoscopic technique was undertaken cautiously only following extensive experience with reduced fluoroscopy protocols. ${ }^{4}$ We realised that ureterorenoscopy could be performed entirely without fluoroscopy in carefully selected patients. We are not the first discipline to omit fluoroscopy from previous image-guided procedures. Interventional cardiologists are now performing cardiac ablations for the correction of atrial fibrillation ${ }^{5}$ and tachycardia using a completely fluoroless technique in children, pregnant women, and obese patients. In gastroenterology, studies have shown that endoscopic retrograde cholangiopancreatography ${ }^{6}$ can be performed without fluoroscopy. Spinal surgeons are now performing craniosacral posterior spinal instrumentation using a fluoroless technique with similar outcomes to the conventional technique. In urology, there have been three previous studies that described a fluoroless technique for ureterorenoscopy. Mandhani et $\mathrm{al}^{7}$ showed that complete 
clearance of distal ureteral stones, below the sacroiliac joint, could be achieved without the use of fluoroscopy in 99 out of 110 patients. Their study also showed that fluoroless balloon dilatation of the distal ureter can also be achieved under direct vision. Tepeler et $\mathrm{al}^{8}$ performed ureterorenoscopy in 93 consecutive patients, avoiding immediate intraoperative fluoroscopy in $92 \%$ of their patients, but obtained a kidney, ureter, and bladder (KUB) film on postoperative day 1 in all patients with an estimated radiation dose of 1.1 millisievert (mSv). In another study, Hsi and Harper ${ }^{9}$ avoided the need for a KUB by using two taps of fluoroscopy at the time of the procedure, thus getting real-time evaluation of stent placement and reducing the median effective dose to $0.05 \mathrm{mSv}$. In their technique, no fluoroscopy was used for the ureterorenoscopy, but limited fluoroscopy was required for stent placement. Although their study continued on the logical progression of dramatically reducing radiation, the study did not provide followup for the patients or any comparison with conventional ureterorenoscopy patients. These previous innovative studies have significantly reduced radiation exposure to patients. Our technique of completely fluoroless treatment of renal and ureteral stones further expands the potential application of fluoroless ureterorenoscopy. Our study is the first to perform mid and distal ureterorenoscopy and stent placement without any form of image guidance. Our series also demonstrates equal stone-free rates between our two cohorts (92\%). Thus, this study demonstrates that a fluoroless technique is feasible and effective for treatment of both mid and lower ureteral stones. One alternative to the use of fluoroscopy during ureterorenoscopy is the use of intraoperative ultrasound. A prospective study by Deters et $\mathrm{al}^{10}$ randomised 50 patients who had been previously stented for symptomatic ureteral stone to either ultrasound or fluoroscopy-guided ureterorenoscopy. There was no difference in stone-free rates, operative time, or complication rates between the two study groups. However, ureteral stents may be difficult to identify using ultrasound. In addition, intraoperative ultrasound requires a unique skill set not possessed by all urologic surgeons. Finally, intraoperative ultrasound may require instruments and personnel, not always available in the operating room. There are certainly some potential benefits of performing a completely fluoroless ureterorenoscopy. Because of the inherent dangers associated with radiation exposure, many institutions have incorporated an ALARA (as low as reasonably achievable) protocol for their medical procedures requiring radiation. We routinely have the fluoroscopy machine available in the room in the event that imaging should be required. With further experience and refinement of techniques, it is possible that eventually the Carm technician and machine would not be required. This would potentially simplify these surgical cases and reduce their cost. Another potential benefit of a fluoroless technique is a reduction in the risk of back pain and other skeletal-related disorders for the surgeon and staff. Surgeons who wear heavy lead aprons for protection from radiation exposure are known to have an increased incidence of spinal complaints.

Ross et al coined the term, interventionalist's disc disease, when they found that cardiologists are at an increased risk of axial skeletal problems related to lead apron use. Interventionalists who wore lead aprons for longer periods of time had a substantially greater incidence of skeletal complaints with more cervical spine ${ }^{11}$ complaints, more multiple-level disc herniations, and more missed days from work due to back pain compared with those wearing aprons for shorter periods of time. Performing procedures in an entirely fluoroless manner means that the surgeon is not required to wear lead shielding to protect against ionising radiation. This study has some limitations. One limitation of this article is that it is retrospective, and therefore unable to describe the patients in whom the fluoroless technique was converted to image guidance. There was also a significant difference in the amount of stone in the groups. Although these differences do exist, both differences make the fluoroless group a clinically more challenging cohort. We continue to revise and adjust our indications for a fluoroless approach as our experience increases. This technique is not appropriate for all patients. Patients who currently would not meet fluoroless criteria in our institution include those with ureteral strictures, entombed stents, significant anatomic abnormalities, and tightly impacted stones. If the surgeons do not feel that they can adequately endoscopically map the entire collecting system to ensure complete fragmentation of all stones, they should not hesitate to employ fluoroscopy. While this study shows favourable patient outcomes in fluoroless ureterorenoscopy, it does not address the additional stresses that may be placed on physicians performing these procedures. Certainly, the fluoroless technique is a significant departure from the conventional technique and it should not be attempted until the surgeon has extensive experience with low radiation protocols for ureterorenoscopy. The intent of this study is not to suggest that fluoroless ureterorenoscopy should be routinely performed in all patients. Rather, the intent of this study was to determine whether ureterorenoscopy without fluoroscopy or other image guidance was feasible and whether it could be safely performed in carefully selected patients. Rigid application of this technique to all patients by surgeons unfamiliar with the technique could result in much harm to the patient. In addition, even experienced surgeons should have a low threshold for employing fluoroscopy if any ambiguity is encountered. Although this cohort of fluoroless ureterorenoscopy was sequential, during this same period some more complicated cases were performed using fluoroscopy, in potential selection bias for the less complicated cases. We strongly recommend that if there is any ambiguity in the case or uncertainty, the surgeon should not hesitate to employ fluoroscopy using a low-dose protocol. Future prospective randomised trials will be needed to delineate optimal patient candidates and to assess the risk-benefit ratio associated with specific patient profiles and surgeon's experience levels. In addition, we will continue to maintain a low threshold for converting to a fluoroscopy-guided technique in the event of any uncertainties or intraoperative concerns.

\section{CONCLUSION}

This series represents the initial description of ureterorenoscopic lithotripsy without fluoroscopy or other form of image guidance performed in the upper tract with a comparison to a cohort of conventional ureterorenoscopy cases. This study demonstrates that ureterorenoscopy without fluoroscopy is technically feasible and can be performed safely in carefully selected patients. 


\section{Abbreviations}

ALARA - As Low As Reasonably Achievable.

BMI - Body Mass Index.

CT - Computed Tomography.

KUB - Kidney, Ureter, and Bladder.

$\mathrm{mSv} \quad$ - Millisievert.

UPJ - Ureteropelvic Junction.

USFDA $^{12}$ - United States Food and Drug Administration.

UVJ - Ureterovesical Junction.

\section{REFERENCES}

[1] Cardis E, Vrijheid M, Blettner M, et al. The 15-Country collaborative study of cancer risk among radiation workers in the nuclear industry: estimates of radiationrelated cancer risks. Radiat Res 2007;167(4):396-416.

[2] Preston DL, Pierce DA, Shimizu Y, et al. Effect of recent changes in atomic bomb survivor dosimetry on cancer mortality risk estimates. Radiat Res 2004;162(4): 377-89.

[3] Georges JL, Livarek B, Gibault-Genty G, et al. Reduction of radiation delivered to patients undergoing invasive coronary procedures. Effect of a programme for dose reduction based on radiation-protection training. Arch Cardiovasc Dis 2009;102(12):821-7.

[4] Greene DJ, Tenggadjaja CF, Bowman RJ, et al. Comparison of a reduced radiation fluoroscopy protocol to conventional fluoroscopy during uncomplicated ureteroscopy. Urology 2011;78(2): 286-90.
[5] Reddy VY, Morales G, Ahmed H, et al. Catheter ablation of atrial fibrillation without the use of fluoroscopy. Heart Rhythm 2010;7(11):1644-53.

[6] Stavropoulos S, Larghi A, Verna E, et al. Therapeutic endoscopic retrograde cholangiopancreatography without fluoroscopy in four critically ill patients using wire-guided intraductal ultrasound. Endoscopy 2005;37(4):389-92.

[7] Mandhani A, Chaudhury H, Gupta N, et al. Is fluoroscopy essential for retrieval of lower ureteric stones? Urol Int 2007;78(1):70-2.

[8] Tepeler A, Armagan A, Akman T, et al. Is fluoroscopic imaging mandatory for endoscopic treatment of ureteral stones? Urology 2012;80(5):1002-6.

[9] Hsi RS, Harper JD. Fluoroless ureteroscopy: zero-dose fluoroscopy during ureterorenoscopic treatment of urinary-tract calculi. J Endourol 2013;27(4):432-7.

[10] Deters LA, Belanger G, Shah 0, et al. Ultrasound guided ureterorenoscopy in pregnancy. Clin Nephrol 2013;79(2):118-23. USFDA. Initiative to reduce unnecessary radiation exposure in patients. 2010. www.fda.gov/Radiation Emitting Products/Radiation Safety/Radiation Dose Reduction/ucm2007191.htm.

[11] Kotil K, Bilge T. Accuracy of pedicle and mass screw placement in the spine without using fluoroscopy: a prospective clinical study. Spine J 2008;8(4):591-6. 